balance by the sole use of exercises, possibly because the weakened muscles are incapable of producing the afferent stimuli necessary to bring about inhibition of the hypertonic antagonists. Graduated muscular contractions induced by means of faradic currents not only exercise the muscle but stimulate the sensory end organs in the muscle, thus artificially supplementing the deficient afferent impulses.

Where nerve injury with axon degeneration accounts for the atony and wasting, the muscles will not respond to faradic stimulation and interrupted galvanic current has to be resorted to.

In some cases re-education of muscle sense and sense of movement is necessary, as, for instance, after tendon transplantation. This may be facilitated in the earlier stages by utilising faradic stimulation of the muscles involved.

As suggested at the beginning of this article, electro-therapy constitutes but a small aspect of rehabilitation, but it is an important one. Many of the methods empioyed are passive ones from the patient's point of view, and therefore they should as soon as expedient be replaced by or combined with more active treatments which should eventually supplant them.

\title{
THE REHABILITATION OF NON-TUBERCULOUS DISEASE AND INJURY OF THE CHEST
}

\author{
By F. S. COOKSEY, M.D.(Lond.) \\ (Medical Officer in charge of rehabilitation, Horton Hospital, E.M.S.)
}

The word rehabilitation has come to be so widely used and diversely applied that it is necessary to clarify the use of the term. Taken in a broad sense it is a convenient omnibus term for a number of positive measures directed towards the restoration of health and social normality. These measures may be enumerated as (I) medical and surgical practice to promote recovery from the effects of disease or injury; (2) ancillary therapeutic methods to assist restoration of function in the disabled part together with the restoration of normal physique in the body as a whole; (3) social organisation to provide freedom from want during illness, adequate domicilary or hospital treatment with eventual return to normal employment; (4) provision for the vocational training of the permanently disabled followed by guaranteed employment, if necessary in sheltered industries.

There is nothing new in the conception or practice of rehabilitation. Indeed the principles are the foundation of good doctoring and as old as the art itself whilst, in the thoracic field, the pioneer work of Papworth and Preston Hall have set a standard exceeded in no other branch. What is new, perhaps, is the effort to co-ordinate the medical and sociological aspects and to apply the principles and practice to the field of general medicine and surgery. The present article is concerned mainly with the application of the ancillary therapeutic methods in the rehabilitation of disease and injury of the chest; but excluding tuberculosis which presents quite separate and highly specialised problems. The ancillary therapeutic methods referred to are physiotherapy, occupational therapy, group exercises and remedial games. Brief reference will be made also to the use of mental exercise or educational therapy.

\section{Breathing exercises.}

Recovery from thoracic illness is dependent, in the first instance, upon the restoration of respiratory function hence it is not surprising that breathing exercises are the predominant therapy.

(a) Inspiratory or lung expansion exercises are used in atelectasis, pneumonia, empyema, after lob- and pneumon-ectomy and as a prophylactic before and after operations on the upper abdomen, etc. Two methods are available; firstly, forced expiration against resistance such as the closed glottis or Wolff's bottles, and secondly, inspiratory breathing exercises. In the former it is difficult to avoid the effort being expended on over-expansion of the normal lung or even the production of emphysema, since the positive pressure is distributed to all parts of the lung and the diseased or damaged area usually offers greater resistance to expansion due to organised fibrinous exudate. In the latter use is made of the negative pressure created by the elevation of the ribs and descent of the diaphragm in normal respiration and the effort is local- 
ised to the collapsed lung by the use of posture and manual pressure to limit movement in the unaffected lung. For example, expansion of the right base is obtained by side bending the trunk to the left with the patient's left hand compressing the left lower ribs and the right arm elevated and crooked over the head so that the stretched latissimus dorsi elevates the right lower ribs. Localisation of effort is further facilitated by a masseuse giving moderate manual counter-pressure on the right lower ribs so that the patient has a resistance to work against. The last mentioned practice makes localised breathing much easier and is helpful in teaching patients to localise inspiratory or expiratory effort to any desired region of the lung. In group exercises at a later stage patients can give counter pressure with their own hand. Under skilful instructors the degree of localisation and range of movement which can be obtained is quite surprising.

Breathing exercises employed alone lead to hyper-ventilation hence general exercises such as skipping and "running on the spot" must be interspersed to create shortness of breath. The preparation of a well balanced and progressive table of exercises calls for skill and experience in the remedial gymnast. To illustrate the reasons for the inclusion of various exercises and the details to which attention must be given the following is a typical example of a table for a case of empyema. The table must provide for localised expansion, mobilisation of the scapula and shoulder on the affected side, prevention or correction of scoliosis, posture training and restoration of the general physique.

I. Skipping to warm up and get short of breath.

2. Deep breathing in all parts of the thorax to improve general respiratory function especially after long illness.

3. Stride jumping with hands clapping above the head to mobilise the ribs and create shortness of breath.

4. Localised breathing with fixation of unaffected lung.

5. Abdominal exercise to reinforce abdominal respiration.

6. Diaphragmatic breathing.

7. Exercise for erector spinae for posture, to elevate the sternum and ribs anteriorly and to correct the tendency to kyphosis resulting from the position which chest patients adopt in bed.

8. Localised breathing.

9. Shoulder loosening exercise.

ro. Deep inspiration and expiration using all parts of chest and diaphragm.

II. Flat foot exercise.

I2. Localised breathing.

13. Trunk turning from side to side with alternate arm flinging for rib and spine mobility and to stretch the pectoral muscles.

I4. Localised breathing.

I5. Final posture correction in front of a mirror.

(b) Expiratory breathing exercises are used in asthma, chronic bronchitis and emphysema. The general handling and approach to these patients is entirely different and success depends less upon technically correct exercises than upon gaining the patients' confidence and co-operation. Before re-education of breathing can be commenced it is necessary to teach the patient to relax the extrinsic muscles of respiration and the whole over-tensed body. The ribs are fixed in the position of forced inspiration and the spine more-or less rigid; so that free mobilising exercises for the thorax should follow relaxation and precede breathing exercises which are directed to diaphragmatic and abdominal breathing, full and prolonged expiration and diminution in upper thoracic respiration. As soon as the breathing at rest is satisfactory the patient must be trained to continue correct breathing whilst performing various activities such as counting aloud, walking up stairs, writing and working in the occupational therapy workshop.

(c) Breathing exercises combined with postural drainage are used in lung abscess and bronchiectasis. Chest manipulations such as hacking, clapping and beating followed by inspiratory and full expiratory breathing in the appropriate position of postural drainage assists the expulsion of pus as a conservative treatment or in preparation for lobectomy; but there is some danger of spreading infection to unaffected parts of the lung so that masseuses should not be allowed to carry out this procedure except under the close supervision of a thoracic physician or surgeon.

(d) Pre-operative breathing exercises are used before lob- or pneumon-ectomy to improve 
the vital capacity of the unaffected lung when the reserve is inadequate; and before thoracic and upper abdominal operations in order that the patient may be able to perform immediate post-operative breathing exercises the more easily.

\section{Massage and Skeletal Exercises.}

Massage for the legs, arms and back is useful for patients with marked muscle wasting after severe toxaemia. Graduated general exercises should follow the massage and special attention should be paid to the postural muscles especially the glutei, quadriceps, abdominals and muscles supporting the arches of the feet. However careful the nursing may be some degree of pes equinus is a common complication of thoracic toxaemia. Too often patients are allowed to get up in low-heeled bedroom slippers and, if pes equinus is present, weight bearing leads to a compensatory pes planus and abduction of the fore-part of the foot. If prophylactic bed exercises are insufficient to correct equinus the heels should be raised an inch or so and the patient instructed to walk with the toes pointed forwards until muscle power and normal range of movement has been restored.

\section{Electrotherapy.}

Heat in the form of radiant heat or diathermy may be used for the relief of pain and to assist the resolution of sepsis. Short wave diathermy has the advantage that electrical contact with the skin is unnecessary and effective heat can be applied to deep seated lesions. Ultraviolet light should be used with caution and is contra-indicated in phthisis. In non-tuberculous cases ultra-violet light is a useful general stimulant in patients who have been ill and hospitalised for a long time; but it is not generally appreciated that patients with toxaemia and especially intra-thoracic infection are more sensitive, as well as less able to respond, to a moderate stimulus than are patients with infection in other parts of the body. It appears to be exposure of the chest wall which is liable to have a deleterious effect so that it is advisable not to irradiate the chest wall until the patient has been observed to be responding well to exposure of ather parts of the body.

The breathing exercises and other forms of physiotherapy briefly described are well established. More recently additional ancillary methods have been developed chiefly due to clinical emphasis upon the value of the early restoration of functional activity, the importance of preventing undue physical and mental deterioration in hospital and the wartime necessity to accelerate the restoration of full working or fighting fitness.

\section{Group Exercises, Physical Training and Games.}

In the early stages individual treatment is necessary because the early institution of posture correction, shoulder movement and breathing exercises is essential; but, at the same time, the patient's general condition and ability to respond to treatment varies so much from day to day that treatment must be adjusted accordingly. As soon, however, as the patient is well enough to start getting up there are many advantages in group exercises. The lively and competitive atmosphere of an exercise class stimulates backward patients and all find exercises less boring than when performed alone. The time consumed in giving all patients individual exercises is such that, inevitably, the tables tend to be hurried or cut short and, consequently, ineffective; whereas a single instructor can give a group of a dozen or more patients an effective table of exercises two or three times a day. In the later stages a short period of exercises once a day is inadequate to restore full function and the capacity for sustained work. A major criticism of past practice is that patients were rendered fit enough to leave hospital; but no provision was made to restore the physique needed for a full working day. The omission can be rectified by providing facilities for sustained exercises and occupational therapy in the hospital rehabilitation centre. Remedial exercises alone become boring when kept up for long periods; thus it comes about that increasing use is made of competitive team games for general strengthening. Volley ball, net ball and basket ball are three games particularly useful in chest work. In short, by the use of group exercises each patient receives more efficient treatment and attains a higher degree of fitness whilst a substantial economy of staff is effected.

The purpose of physical treatment is to encourage and lead the patient to making a greater effort from day to day. To be effective exercise tables must be continuously progressive in power and duration. For convenience in prescribing patients may be grouped into four stages 
or grades. The physician can prescribe the grade of exercises suitable for the individual patient and leave to the instructor the detailed progression of exercises within each grade. Four convenient grades are (I) individual exercises for the bedridden; (2) easy or "sitting" group exercises for those just out of bed; (3) strong or "standing" group exercises for the fully ambulant and (4) physical training and games for general hardening in the final stages.

An interesting recent development is the realisation that the presence of a correctly placed and fitting drainage tube does not prevent the patient playing games and performing strenuous exercises. Patients with empyema and a drainage tube can be in the sitting class within a week of operation, in the standing class at a fortnight and playing volley ball at three weeks or a month. The improvement in physique and speed of obliteration of the cavity can be ascribed to sound surgery permitting the early restoration of vigorous functional activity.

By no means all patients will tolerate rapid progression of exercises. Chest patients are particularly prone to exacerbations of infection and associated toxaemia so that the cardinal rule is that exercises must be graded to evoke an optimal response and adjusted to the day by day condition of the patient.

\section{Occupational Therapy.}

(a) Diversional therapy consists in the use of various handicrafts, interesting in themselves as hobbies, to prevent boredom, introspection and loss of functional activity in patients who are hospitalised for long periods. The work can be carried out in bed or in a handicrafts room and is particularly useful in thoracic centres. Except for the shortage of most materials in wartime the variety of handicrafts is so wide that there must be few patients who cannot be found something to interest them. Patients should be allowed to keep their work for the cost of the materials used and the occupational therapist must see that the work selected is within the patient's capacity to ensure pride in the finished article.

(b) Remedial therapy makès use of various crafts with tools suitably modified to encourage the restoration of specific movement, muscle power and function. This section, which is of particular value in traumatic surgery, has limited application in thoracic work. Certain forms of weaving and basketry are useful for stiff shoulders and tight pectorals. Gardening is useful to get chest patients out of doors in fine weather and carpentry is useful as a supplement to physical training in the final stages of hardening; but no present forms of occupational therapy are of value to improve respiratory function.

(c) Vocational training of the permanently disabled to suitable alternative trades within the capacity of the individual is outside the scope of hospital work; but arrangements should be made for patients likely to need such training whilst they are still under treatment so that there is no delay. Furthermore, the knowledge that the future is provided for is of obvious psychological value whilst the patient is still in hospital.

\section{Educational Therapy.}

The sedentary worker such as the clerk, typist, storekeeper and cashier is apt to resent physical training and occupational therapy on the grounds that they need mental capacity rather than good physique or manual dexterity. After long and debilitating illness the clerical worker usually complains of headache and inability to concentrate after an hour or two. For the rehabilitation of this type of worker it is necessary to provide means to improve the power of mental concentration and endurance. This can be achieved by graduated mental exercise in the same way that physical exercise and occupation is provided for the manual worker. Essential remedial exercises to improve thoracic function must be carried out, but the advanced physical training and occupational therapy can be replaced by organised study. In practice it is desirable to include some mental exercise for the labourer and some physical training for the clerk; but the major effort will be adjusted to the type of work for which the patient has to be rendered fit.

Mental exercise is provided by discussion groups on subjects of general or contemporary interest, lectures on subjects of general interest, short talks prepared and given by patients, correspondence courses for young patients. Draughts, chess, cards and other games organised in tournaments to cover increasing periods of time. Refresher courses in shorthand, arithmetic, etc. Play reading and the provision of library books.

In conclusion the aim of the rehabilitation centre may be summarised as, firstly, the pro- 
vision of specific remedial physiotherapy for a short period a day in the early stages with the progressive addition during convalescence of physical training, occupational therapy and mental exercise so that gradually the whole day is devoted to the process of recovering the physical and mental capacity for a full day's work. For example a patient in the later stages of convalescence from a severe pneumonia might spend the final days in hospital in the following manner. 9-Io ward duties, I0-I0.30 standing chest class, I0.30-II break, II-I2 lecture, I2-I lunch, I-3 workshops or gardening, 3-4 standing chest class followed by games, 4 tea. In this manner a few days extra are spent in hospital in getting thoroughly fit instead of leaving at the earliest possible moment and spending an indeterminate convalescence at home. It is not necessary to live in hospital for the final stages and, indeed, the greater contentment of being able to live at home is a positive contribution to rehabilitation; but facilities must be available for the patient to spend the whole day at the rehabilitation centre and to have a midday meal and rest.

\section{SURGERY IN OLD AGE}

\section{By D. LANG STEVENSON, F.R.C.S. (Assistant Surgeon, Essex County Hospital, Romford)}

The ability of an old person to stand an operation is not infrequently underestimated because undue significance is given to numerical age, and the tendency to let this play an over important part in the assessment of stamina denies some of the more tough a reasonable chance of cure or relief from some distressing complaint.

Just as old age bears no constant relation to decline in physical fitness or mental acuity, in the same way it is no reliable measure of operability. In the evening of human lifetime strong contrasts become apparent, distinguishing those who have treated their bodies well and who possess the inherited longevity of sound stock from those who have abused health, or who have been unfortunate in their inheritance. An example of this is often seen in the " prostate ward," where an alert fresh-complexioned octogenarian has a companion in local pathology a veritable human derelict who may be fifteen years the junior. It is therefore important both to recognise that those old patients bearing testimonials of long healthy lives, similarly enjoyed by their parents, have remarkable powers of tolerating surgical trauma, and also that those of poorer fibre can be more often escorted safely through surgical illness if the management of their case is modified to suit the needs of frail old age.

Frank evidence of inadequate powers of healing or resistance to infection is not frequently seen in old patients, but there is little doubt that tissue vitality is reduced by a variable degree of cellular atrophy and diminution in the quantity and quality of the blood supply. The same measures which may be effective in making good some of these deficiencies in the vitality of ageing tissues have a considerable value in preventing the post-operative complications, pulmonary infection or oedema, thromboses, and renal failure, peculiar to those advanced in years.

The widespread ill-effects which Penton, Stephenson and Korovensky (I94I) have shown may be caused by deficiency of vitamin $B$ and $C$ in old persons, and the important part that vitamin C (Hunt,.I940) plays in wound healing cân leave no doubt as to the advisability of giving adequate amounts of these vitamins before and after operation. In the opinion of these workers senile vitamin deficiency is not only caused by the low vitamin diet common amongst old people, but is also influenced by defective absorption. They therefore recommend large therapeutic dosage. Tissue anoxia favoured by emphysematous lungs within a fixed thorax and a sluggish circulation within a rigid arterial system can be remedied to a worth-while extent by maintaining bodily movements in the form of regular limb and breathing exercises, and by inhalations of oxygen through a B.L.B. mask during the early post-operative period. Helping these old patients out of bed into a chair on the day following operation insists on a valuable increase in respiration and circulation rate. Stasis can be further prevented by careful attention to water balance, and in certain cases by the controlled administration of thyroid extract. The latter has the noticeable effect of livening up some of these old patients as if the stimulation to metabolism was shared by the waning endocrine system.

The cost of heparin prohibits its general use, but in selected cases prophylactic administration during the first post-operative week is an additional safeguard. 